\title{
CS Research Square \\ Health problems and general anxiety among traffic police working in two major cities of Nepal
}

Subash Thapa ( $\nabla$ soobesh@gmail.com )

https://orcid.org/0000-0002-1182-8511

\section{Sargam Ghimire}

Pokhara University

Arja R Aro

Syddansk Universitet

\section{Research note}

Keywords: Air pollution, General anxiety, Cross-sectional study, Occupational health problems, Traffic police, Nepal

Posted Date: July 4th, 2019

DOI: https://doi.org/10.21203/rs.2.10928/v1

License: (c) (i) This work is licensed under a Creative Commons Attribution 4.0 International License. Read Full License 


\section{Abstract}

Objective Traffic police are at-risk of various health problems due to an exposure to air pollution and high traffic; and traffic roads in some Nepalese cities are very congested. There is very little information about health problems of Nepalese traffic police. Therefore, a cross-sectional, self-administered questionnaire study among 252 traffic police was conducted to identify the common health problems and factors associated with different levels of general anxiety among traffic police working in two major cities of Nepal. Results Around $55 \%$ of traffic police had ear ringing problem, $57 \%$ had burning eyes, $64 \%$ had back pain, and $67 \%$ had breathing difficulties in the previous three months. Around $33 \%$ had moderate anxiety and $23 \%$ had persistent and severe anxiety. The multinomial logistic regression analysis showed that job dissatisfaction, ear ringing and eye irritation in the previous three months and having physical symptoms, namely eye irritation, ear ringing and back pain in last three months, were significantly associated with moderate anxiety. Further, age (25-30 years), gender (female), job dissatisfaction, and reporting ear ringing symptoms in the previous three months were significantly associated with severe anxiety.

\section{Introduction}

Traffic police, who are responsible for overseeing traffic safety compliance on roads, are at risk of various health problems including musculoskeletal disorders and non-communicable diseases [1 2]. Some studies [3-6] have reported that occupational factors, such as job strain, work conflicts, climate, health problems, exposure to threats and violence, are associated with mental health problems, including general anxiety. Most of these studies have been conducted in a developed country context; however, such studies on occupational health problems of traffic police working in highly polluted areas in developing countries, are also important to draw attention of the local government and the public to this important issue.

In Nepal, transportation has become more and more disturbing and dangerous over the years, and the problem of traffic congestion and work surroundings can be deemed as a major issue affecting health and wellbeing, especially of traffic police personnel. Especially in the Nepalese major cities such as Kathmandu and Lalitpur, the automobile exhaust and vehicular emissions from poorly maintained vehicles along with large increase in number of automobiles and the congestion of traffic, have created an alarming air pollution problem [7]. Majumder et al. (2012) have noted that the occupational and ambient PM10 concentrations in high density traffic area and road intersection in Kathmandu and Lalitpur greatly exceeded the $24 \mathrm{~h}$ average limit value (The ambient air quality $=120 \mu \mathrm{g} \mathrm{m}-3$, which exceeds by $2-3$ folds the level recommended by international guidelines) [7]. Traffic police in Kathmandu and Lalitpur work within the close proximity to the vehicles and congested roads standing for several hours and are exposed to high levels of air and noise pollution in a regular manner, which may lead to respiratory diseases, reduced lung function, noise-induced hearing loss and other health problems [8-10].

In addition, traffic police are highly stressed due to their nature of work, work conditions and work surroundings, and a number of these occupational factors could attribute to general anxiety among 
traffic police [11]. There have no studies documented yet explaining health problems including general anxiety among Nepalese traffic police. Therefore, in this study, our aim was to identify the common health problems and the factors associated with general anxiety among the traffic police working in Kathmandu and Lalitpur. We believe, this sort of occupational health studies would help us to understand occupational circumstances and their adverse influence on health and wellbeing of traffic police [11].

\section{Methods}

This was a community-based, cross-sectional, self-administered questionnaire study conducted among the traffic police, working in Kathmandu and Lalitpur in 2015.

Sampling and recruitment: Traffic police who were deputed in the busy roads were recruited in the study and those who worked in a non-field environment (e.g., administrators, office assistants, and drivers) were excluded. Of the total 32 traffic units in Kathmandu and Lalitpur, 10 traffic units were randomly selected, and a total of 260 traffic police from these selected traffic units were invited to participate in the study. The sample size was calculated taking 0.5 as proportion of traffic police with (some level of) anxiety, $5 \%$ as an allowable error and 1,175 as the reported total number of traffic police who were working in Kathmandu and Lalitpur [12].

Questionnaire and measurement: The questionnaire covered socio-demographic information, occupationrelated information, self-reported physical health problems and presence of general anxiety. A written consent procedure was completed before the self-administration of the questionnaire.

General anxiety was assessed using the Beck Anxiety Inventory (BAl) that is a 21-item self-report inventory of the severity of anxiety symptoms (e.g., feeling hot, fear of losing control). Participants were asked to rate how much each symptom bothered them 'during the past week, including today' on a 4point-rating scale ranging from 'not at all' (0) to 'severely-I could barely stand it' (3). The possible range of the total score is from 0 to 63. According to the BAl cutoff-scores used by Rudaz et. al. (2017), the scores between 0 and 21 indicated low anxiety, scores between 22 and 35 indicated moderate anxiety, and scores of 36 and above indicated severe anxiety [13 14]. For non-clinical use, the cut-off scores may vary [15]. In the current sample, the BAI had good internal consistency (Cronbach's $a=.85$ ).

Data analysis: Descriptive statistics comparing frequencies and percentage between subgroups was calculated and multinomial logistic regression analyses as a multivariate approach to assess the factors associated with general anxiety was used. For the multinomial regression analyses the above-mentioned anxiety levels of low, moderate and severe were used as the outcome variable and 'no or very low-level anxiety' was taken as reference category. Religion was not included in the multivariable analysis due to low number in some cells. All analyses were conducted with PASW 22.0 (SPSS, Inc. Chicago, IL) and p < 0.05 was considered statistically significant.

\section{Results}


We approached 260 traffic police; 8 (male) traffic police did not consent to participate and thus, the study comprised of 252 traffic police (response rate $=97 \%$ ).

Socio-demographic characteristics: Mean age of the respondents was 27.7 years (Standard Deviation=4.6 year). Most of the respondents were male (90.1\%), Hindu by religion ( $95.2 \%)$ and had completed higher secondary or above education (69.8\%) (see Table 1).

More than half of the respondents had been working for more than five years (58.3\%) and had an average work shift of 11 hours or more (74.6\%). One-third of the traffic police did not routinely use a protective equipment (e.g., masks, eye glasses) during their work (34.4\%), and the commonly cited reasons for the non-use were discomfort, and headache and dizziness associated with the equipment use.

Around $20 \%$ of the traffic police were dissatisfied with their job and $42.7 \%$ had accidents at work. Only $34.5 \%$ had a periodic health examination visit within the previous year, and the most cited reason for not having a periodic medical check-up was the lack of time due to long working hours.

Self-reported health problems among the traffic police: Among the total traffic police, $55.0 \%$ had ringing ear symptoms, $34.5 \%$ had irritation in their eyes, $64.3 \%$ had back pain due to prolong standing and $66.7 \%$ had breathing difficulty in the previous three months. Altogether 110 respondents (43.7\%) had no anxiety or low-level anxiety, $84(33.0 \%)$ had moderate anxiety and $58(23.0 \%)$ had persistent or severe anxiety.

Table 1. Socio-demographic and occupational characteristics of the traffic police

The adjusted multivariable analysis showed that job dissatisfaction and having physical symptoms, such as eye irritation, ear ringing and back pain in last three months, were significantly associated with moderate anxiety. Further, age (25-30 years), gender (female), job dissatisfaction, and having ear ringing symptom in the previous three months were significantly associated with severe anxiety (see Table 2).

Table 2. Associations between socio-demographic and occupational variables and moderate and severe anxiety among the traffic police $(\mathrm{N}=252)$

\section{Discussion}

Occupational health hazards of the traffic police could get more severe when the duration of exposures to physical strain, exhaust of vehicles, noisy environment due to blowing of horns and blow of dust in the air by a speeding vehicle, increase [16-18]. This was supported by the fact that age, a proxy of duration of work, was significantly associated with severe anxiety among the traffic police in our study.

The relatively low use of protective equipment found in this study can contribute to high burden of occupational health problems among traffic police [18 19]. Studies have shown a significant protective effect of using N95 mask from several respiratory health problems and infections [20 21]. Among Nepalese traffic police, the low use of such protective equipment could be a consequence of poor knowledge or low intention even with adequate knowledge [4 9]. A study (2015) reported that the level of 
knowledge regarding the prevention of respiratory problems among Nepalese traffic police was significantly higher than the use [9]. Since the preventive practice is poor, and the burden of self-reported health problems is high, the government should strongly mandate the use of protective equipment during the work to improve the level of preventive practice among traffic police.

In this study, a significant proportion of traffic police had never accessed health screening services and reported having various physical and mental health symptoms. For instance, over $65 \%$ of the traffic police reported having back pain; this may be because a clear majority of traffic policemen reported working in standing position an average of 11 hours a day. In addition, having symptoms such as eye irritation and ear ringing are clearly linked with the air pollution and congested traffic, and reporting these symptoms was also significantly associated with moderate anxiety among the traffic police in the study. Government of Nepal, however, does not recognize the occupational health of traffic police as a major public health issue and there are no specific health services that are provided to traffic police to protect them from potential occupational health problems and injuries.

It has been reported in online-media (Facebook page of Nepal Health Clinic Association) that sporadic health camps were being organized by non-governmental organizations targeting traffic police; however, most traffic police, especially females and those working long shifts, are less likely to participate in those health camps. Moreover, these camps may not have all the services and resources required to comprehensively screen for all the occupation-related physical and mental health problems among traffic police. It is recommended that a special occupational health taskforce under Ministry of Health and Population of Nepal should be established and mobilized to target traffic police with the tailored occupational health screening services (e.g., monitoring exposure to environmental toxicants, noise, accidents, communicable diseases and mental stress).

Unless the occupational surroundings (air pollution, noise pollution and traffic congestion) are not made healthier, the health and safety of traffic police is less likely to be insured [8 16 18]. It is therefore suggested that pollution control and traffic management policies and strategies are of utmost importance and should be effectively implemented to ameliorate occupational health and wellbeing of traffic police working in Kathmandu and Lalitpur. The traffic management authorities in Kathmandu and Lalitpur have introduced several rules and strategies (e.g., banning and removing vehicles that are 20 years and older, mandatory use of filter for gas exhaustion, introduction of electrically powered vehicles, improvement in traffic management) to tackle the problems of traffic congestion and air pollution; however, the effective implementation of such rules and strategies has become a challenging task. Further research on implementation issues of how and why such polices are not producing desired (health and traffic-related) outcomes could come up with insightful conclusions [11].

\section{LIMITATIONS}

In this study, we approached and invited 260 traffic policemen and we received a high response rate (97\%), given that traffic police in Kathmandu and Lalitpur usually have long shifts and tiring nature of work. We also used a standard instrument to measure general anxiety, which increases comparability of 
our findings with other studies and prevented misclassification of information. Nevertheless, the following limitations ought to be considered when interpreting the results: first, since we relied on selfreported data and no medical tools were used to assess health problems systematically, it appears likely that the higher prevalence of occupational health problems among traffic police might be due to reporting and recall bias. Second, the cross-sectional nature of the study prohibits inference of causality. Third, we did not consider other social and environmental factors that can potentially be associated with health problems of traffic police. Forth, research has demonstrated that the ordinal categorization of continuous constructs, such as anxiety, may lead to less reliable measurement [22]. Last, gender was significantly associated with severe anxiety despite the very low number of female traffic police included in the study. In fact, the representation of women in traffic police department of Nepal is very low and therefore, this finding should be cautiously interpreted.

\section{List Of Abbreviations:}

BAI= Beck Anxiety Inventory

PM10= Particulate matter 10

SPSS $=$ Statistical Package for the Social Sciences

\section{Declarations}

Ethics approval and consent to participate: The Ethical Review Board of Nobel College Pokhara University provided ethical clearance for the study (Ref. No. 08/014). Written informed consent was taken from all the respondents.

Consent for publication: Not applicable

Availability of data and material: The dataset during and/or analyzed during the current study available from the corresponding author on reasonable request.

Competing interest: The author(s) declare that they have no competing interests.

Funding: No specific funding was received with regards to this manuscript

Authors' contributions: ST and SG led the design of the study, and SG conducted the field work. ST analyzed the data and developed the first draft of the manuscript. All authors reviewed and approved the final draft.

Acknowledgements: The authors would like to thank the District Traffic Police Office of Kathmandu and Lalitpur, Nepal for their invaluable support and help during the field work of the study.

\section{References}


1. Satapathy D, Behera T Fau - Tripathy R, Tripathy R. Health status of traffic police personnel in brahmapur city. Indian J Community Med 2009;34(1):71-2 doi: D - NLM: PMC2763644 EDAT- 2009/10/31 06:00 MHDA- 2009/10/31 06:01 CRDT- 2009/10/31 06:00 AID - 10.4103/0970-0218.45380 [doi] PST ppublish[published Online First: Epub Date]|.

2. Sharif A, Taous A Fau - Siddique BH, Siddique Bh Fau - Dutta PG, Dutta PG. Prevalence of noise induced hearing loss among traffic police in Dhaka Metropolitan City. Mymensingh Med J 2009;18(1):24$28 \mathrm{~S}$

3. Cattaneo A, Taronna M, Consonni D, Angius S, Costamagna P, Cavallo DM. Personal exposure of traffic police officers to particulate matter, carbon monoxide, and benzene in the city of Milan, Italy. J Occup Environ Hyg 2010;7(6):342-51 doi: 10.1080/15459621003729966[published Online First: Epub Date]|.

4. Kroenke K, Outcalt S Fau - Krebs E, Krebs E Fau - Bair MJ, et al. Association between anxiety, healthrelated quality of life and functional impairment in primary care patients with chronic pain. Gen Hosp Psychiat 2013;35(4):359-65 doi: 10.1016/j.genhosppsych.2013.03.020[published Online First: Epub Date]|.

5. van der Vaart R, Weaver MA, Lefebvre C, Davis RM. The association between dry eye disease and depression and anxiety in a large population-based study. Am J Ophthalmol 2015;159(3):470-74 doi: 10.1016/j.ajo.2014.11.028[published Online First: Epub Date]|.

6. Wieclaw J, Agerbo E Fau - Mortensen PB, Mortensen Pb Fau - Burr H, Burr H Fau - Tuchsen F, Tuchsen F Fau - Bonde JP, Bonde JP. Psychosocial working conditions and the risk of depression and anxiety disorders in the Danish workforce. BMC Public Health 2008;8(8):280 doi: D - NLM: PMC2519085 EDAT2008/08/09 09:00 MHDA- 2008/09/20 09:00 CRDT- 2008/08/09 09:00 PHST- 2007/12/17 [received] PHST- 2008/08/07 [accepted] PHST- 2008/08/07 [aheadofprint] AID - 1471-2458-8-280 [pii] AID 10.1186/1471-2458-8-280 [doi] PST - epublish[published Online First: Epub Date]|.

7. Majumder AK, Nazmul Islam KM, Bajracharya RM, Carter WS. Assessment of occupational and ambient air quality of traffic police personnel of the Kathmandu valley, Nepal; in view of atmospheric particulate matter concentrations (PM10). Atmospheric Pollution Research 2012;3(1):132-42 doi: https://doi.org/10.5094/APR.2012.013[published Online First: Epub Date]|.

8. Shrestha I, Shrestha BI Fau - Pokharel M, Pokharel M Fau - Amatya RCM, Amatya Rc Fau - Karki DR, Karki DR. Prevalence of noise induced hearing loss among traffic police personnel of Kathmandu Metropolitan City. Kathmandu Univ Med J 2011;9(36):274-78

9. Aryal Bhandari A, Gautam R, Bhandari S. Knowledge and Practice on Prevention of Respiratory Health Problems among Traffic Police in Kathmandu, Nepal. International Scholarly Research Notices 2015;2015:6 doi: 10.1155/2015/716257[published Online First: Epub Date]|. 
10. Devare Phadke S, Patra P, Iqbal R. Psychosocial Problems in Traffic Police:Cross Sectional Survey Using COPSOQ. Scholars Journal of Applied Medical Sciences (SJAMS) 2014;2(6G):3380-82

11. Patil RR, Chetlapally SK, Bagavandas M. Global review of studies on traffic police with special focus on environmental health effects. International journal of occupational medicine and environmental health 2014;27(4):523-35 doi: 10.2478/s13382-014-0285-5[published Online First: Epub Date]|.

12. Lemeshow S, Hosmer Dw Jr Fau - Klar J, Klar J. Sample size requirements for studies estimating odds ratios or relative risks. Stat Med 1988;7(7):759-64

13. Beck AT, Epstein N, Brown G, Steer RA. An inventory for measuring clinical anxiety: psychometric properties. Journal of consulting and clinical psychology 1988;56(6):893-7

14. Rudaz M, Ledermann T, Margraf J, Becker ES, Craske MG. The moderating role of avoidance behavior on anxiety over time: Is there a difference between social anxiety disorder and specific phobia? PLoS ONE 2017;12(7):e0180298 doi: 10.1371/journal.pone.0180298[published Online First: Epub Date]|.

15. Beck A, Steer R. Beck Anxiety Inventory Manual. . San Antonio, Texas: Psychological Corporation, 1993.

16. Kaliaganov Pi Fau - Troshin VV, Troshin Vv Fau - Makarov IA, Makarov IA. Influence of working environment factors on health state of road police officers controlling traffic (review of literature). Med Tr Prom Ekol 2009;5:30-35

17. Karita K, Yano E Fau - Tamura K, Tamura K Fau - Jinsart W, Jinsart W. Effects of working and residential location areas on air pollution related respiratory symptoms in policemen and their wives in Bangkok, Thailand. Eur J Public Health 2004;14(1):24-26 doi: DOI 10.1093/eurpub/14.1.24[published Online First: Epub Date]l.

18. Li YC, Huang Hj Fau - Zhang Z-I, Zhang ZI Fau - Qi X-y, Qi XY. Effects of occupation on health of traffic policemen in a city. Zhonghua Lao Dong Wei Sheng Zhi Ye Bing Za Zhi 2008;26(3):165-7

19. Lesage FX, Jovenin N Fau - Deschamps F, Deschamps F Fau - Vincent S, Vincent S. Noise-induced hearing loss in French police officers. Occup Med (Lond) 2009;59(7):483-6 doi:

10.1093/occmed/kqp091[published Online First: Epub Date].

20. Mukerji S, Maclntyre CR, Newall AT. Review of economic evaluations of mask and respirator use for protection against respiratory infection transmission. BMC Infectious Diseases 2015;15:413 doi: 10.1186/s12879-015-1167-6[published Online First: Epub Date]|.

21. Maclntyre CR, Wang Q, Rahman B, et al. Efficacy of face masks and respirators in preventing upper respiratory tract bacterial colonization and co-infection in hospital healthcare workers. Preventive Medicine 2014;62:1-7 doi: https://doi.org/10.1016/j.ypmed.2014.01.015[published Online First: Epub Date]|. 
22. Markon KE, Chmielewski M, Miller CJ. The reliability and validity of discrete and continuous measures of psychopathology: a quantitative review. Psychological bulletin 2011;137(5):856-79 doi: 10.1037/a0023678[published Online First: Epub Date]|.

\section{Tables}

Table 1. Socio-demographic and occupational characteristics of the traffic police 


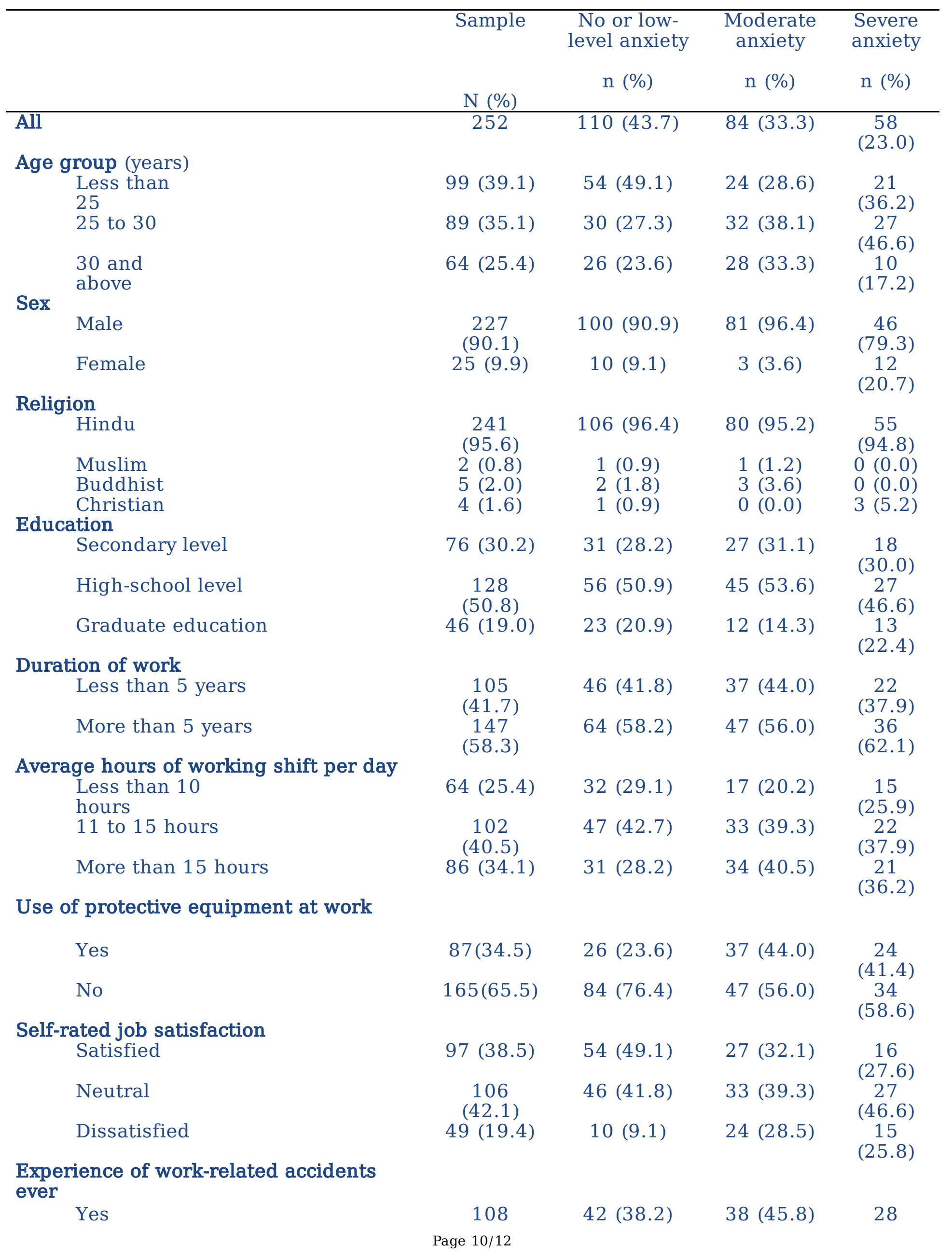


No
dic medical examination visit during
revious year

Periodic medical
the previous year
Yes

the pres

No

Ear ringing during the previous three months

Yes

No

Irritation in the eyes during the previous three months

Yes

No

Back pain during the previous three months Yes

No

Breathing difficulties during the previous three months

Yes

No
$(42.7)$

142

(56.1)

68 (61.8)

45 (54.2)

(49.1)

29

(50.9)

$\begin{array}{cccc}165 & 32(29.1) & 17(20.2) & 17 \\ (65.5) & & & (29.3) \\ 87(34.5) & 78(70.9) & 67(79.8) & 41 \\ & & & (70.7)\end{array}$

138

(55.0)

113

(45.0)

37 (33.6)

59 (70.2)

73 (66.4)

25 (29.8)

(73.3)

15

(26.3)

87 (34.5)

165

(65.5)

63 (57.3)

78 (92.9)

$6(7.1)$

47 (42.7)

(86.2)

8 (13.8)

162
$(64.3)$
$90(35.7)$

81 (73.6)

49 (58.3)

29 (26.4)

35 (41.7)

(53.2)

26

(44.8)

$$
\begin{gathered}
168 \\
(66.7)
\end{gathered}
$$

$84(33.3)$
$66(60.0)$

$44(40.0)$
61 (72.6)

23 (27.4)
41 (70.7)

17 (29.3)

Table 2. Associations between socio-demographic and occupational variables and moderate and severe anxiety among the traffic police $(\mathrm{N}=252)$ 
Age group (years)

$$
\text { Less than } 25
$$

25 to 30

30 and above

Gender

Female

Male

Job satisfaction

Dissatisfied

Neutral

Satisfied

Ear ringing during the previous three months Yes

No

Irritation in the eyes during the previous three months

Yes

No

Back pain during the previous three months

Yes

No

AOR, Adjusted Odds Ratio; ${ }^{*} \mathrm{P}<0.05$
$0.662(0.279-1.573) \quad 0.981(0.345-2.791)$

$1.203(0.514-2.817) \quad 2.863(1.054-$

7.775)*

1 (Reference)

1 (Reference)
$4.019(1.129-$

$14.312)^{*}$

1 (Reference)

1 (Reference)
$3.586(1.150-$

$$
\text { 9.203)* }
$$

$1.351(0.622-2.936) 1.643(0.694-3.888)$

1 (Reference)

1 (Reference)

$2.831(1.371-$

$5.848)^{*}$

1 (Reference)
$4.955(2.142-$ $11.458)^{*}$

1 (Reference) $4.162(1.513-\quad 1.730(0.645-4.641)$ $11.446)^{*}$

1 (Reference)

$4.164(1.202-$ $14.423)^{*}$

1 (Reference)
$2.118(0.635-7.061)$

1 (Reference) 